\title{
Polymer dynamics in linear mixed flow
}

\author{
Arti Dua \\ Max Planck Institute for Polymer Research, P.O. Box 3148, D-55021 Mainz, Germany \\ Binny J. Cherayil \\ Department of Inorganic and Physical Chemistry, Indian Institute of Science, Bangalore-560012, India
}

\begin{abstract}
Recent simulations by Chu et al. [Phys. Rev. E 66, 011915 (2002)] on the behavior of bead-spring and bead-rod models of polymers in linear mixed flows (flows with unequal amounts of extension and rotation) are compared with the predictions of a finitely extensible Rouse model that was used earlier [J. Chem. Phys. 112, 8707 (2000)] to describe the behavior of long flexible molecules of $\lambda$-phage DNA in simple shear. The model is a generalization of the continuum Rouse model in which the "spring constant" of the bonds connecting near neighbor segments is allowed to become nonlinearly flow-dependent through a term involving the initially unknown mean square size of the chain, $\left\langle\mathbf{R}^{2}\right\rangle$. A self-consistent equation for this quantity is derived by using the flow-modified Hamiltonian to calculate it from its statistical mechanical definition. After solving this equation numerically, the mean fractional extension of the chain $x$ can be obtained as a function of the Weissenberg number Wi and a mixing parameter $\alpha$. The results compare favorably with data from the simulations of Chu et al., and suggest the existence of a scaling variable $\mathrm{Wi}^{\mathrm{e} f f}=\sqrt{\alpha} \mathrm{Wi}$ in terms of which separate curves of $x$ versus Wi fall more or less on a single universal curve.
\end{abstract}

\section{INTRODUCTION}

Single molecule studies have shown over the last few years that the response of long chain molecules to the effects of even simple types of flow can be quite dramatic. ${ }^{1-5}$ This is especially true of simple shear, where the polymer can not only stretch out in the shape of a long thin rod, but can also exist-transiently-in states of more unusual geometry, including dumbbells, half-dumbbells, and fish hooks. ${ }^{1}$ Such states are in general the product of the dynamic interplay between the flow's rotational and elongational components, which, for simple shear, are exactly equal to each other. In this respect, simple shear can be regarded as a "critical" flow, ${ }^{6}$ one where the amount of vorticity just prevents the chain from making a transition to a completely extended configuration. Like equilibrium critical phenomena, simple shear is distinguished by the existence of large and longlived fluctuations, in this case fluctuations in the size of the chain. These fluctuations are responsible for the chain's myriad conformational states, which can actually be captured on film, ${ }^{1,2}$ or identified in computer simulations. ${ }^{7-11}$

To probe the immediate neighborhood of this "critical point," Chu and co-workers have recently simulated the dynamics of bead-rod and bead-spring polymers that are subject to so-called linear mixed flows. ${ }^{12}$ These are flows where the amounts of vorticity and strain rate are no longer exactly the same,$^{13}$ the excess or deficit of one of these components with respect to the other being characterized by a parameter $\alpha$. In the limit $\alpha \rightarrow 0$, the flow reduces to simple shear, while the limits $\alpha \rightarrow 1$ and $\alpha \rightarrow-1$ correspond to pure elongational and pure rotational flow, respectively. By studying the behavior of the chain when $\alpha$ is small but not exactly 0 , Chu and co-workers have been able to study the onset of the instabili- ties that cause the large fluctuations that occur in simple shear. Their results, expressed in terms of the fractional extension $x$ vs the Weissenberg number Wi at different fixed $\alpha$, provide a detailed map of the relative importance of the elongational component in producing extended chain configurations. Significantly, when $x$ is replotted against an effective flow parameter, defined as $\mathrm{Wi}^{\text {eff }} \equiv \mathrm{Wi} \sqrt{\alpha}$, the data are all found to fall approximately on the same universal curve. The existence of a scaling relation of this kind is what one would expect from a phenomenon that seems to share attributes with a critical point. ${ }^{14}$

In two earlier analytic theories of the polymer-flow problem, ${ }^{15,16}$ we showed that the Rouse model of chain dynamics is unable to provide a satisfactory description of experimental data ${ }^{1}$ (also from the Stanford group) on dilute solutions of flexible DNA molecules in simple shear. The deficiencies of the model, which can be traced to the Gaussian statistics that allow unphysical extensions of the chain beyond its contour length, ${ }^{17}$ were corrected by introducing a constraint on the maximum permissible chain extension at high rates of shear, with significant improvements to the degree of agreement between theory and experiment. Two different ways of imposing the constraint were considered: one approach leads to a variant of the finitely extensible nonlinear elastic ("FENE") model, ${ }^{18}$ while the other leads to a variant of the Kratky-Porod wormlike model. ${ }^{19}$ The latest studies on polymers in mixed flows provide additional data that can be used to test the utility of such "constrained" Rouse models in more general situations. The basic objective of this paper is therefore to compare the predictions of the FENE-type model with these data. We are also interested in a similar comparison using the Kratky-Porod-type model, but 
the required calculations are nontrivial, and will therefore be left for future work.

The following section reviews the salient features of the finitely extensible Rouse model. The dynamics of the model under the action of linear mixed flow is assumed to be governed by a Langevin equation, whose solution is obtained in Sec. III using Laplace transforms. The solution of the flow equation is used to derive an equation for the steady state mean square end-to-end distance of the chain, which is solved numerically for different values of the strength of the flow (expressed in terms of the Weissenberg number Wi) and the degree of mixing $\alpha$. The results are presented as plots of the mean fractional extension $x$ versus Wi for various fixed values of $\alpha$, and are discussed in Sec. IV.

\section{THE FINITELY EXTENSIBLE ROUSE MODEL}

The equilibrium conformation of a polymer, in the absence of excluded volume interactions, can be described as a chain of $n$ beads joined by springs of strength $b$ that are separated from each other by an average bond distance $l .^{20}$ In the limit $n \rightarrow \infty, l \rightarrow 0, n l \rightarrow N$, this bead-spring model of the polymer describes a continuous curve whose Hamiltonian $H$ (in units where the thermal energy $k_{B} T$ is 1 ) is given by

$$
H=\frac{3 b}{2} \int_{0}^{N} d \tau\left(\frac{\partial \mathbf{r}(\tau)}{\partial \tau}\right)^{2}
$$

where the variable $\mathbf{r}(\tau)$ is the vectorial distance, with respect to some Cartesian coordinate system, of a point on the curve that is a distance $\tau$ from one end of the chain.

For a free polymer (no external force field), the spring constant $b$ is $1 / l$, and Eq. (1) is then equivalent to the wellknown Wiener representation of the random walk. ${ }^{21}$ If there are forces on the chain (as a result of flow, for instance), its Hamiltonian is no longer given by Eq. (1), but Eq. (1) can nevertheless serve as an approximation to the exact $H$ provided $b$ is allowed to depend on the external forces. In general, the dependence of $b$ on these forces cannot be determined a priori, but it is possible to suggest plausible functional forms for it from physical considerations. Following the model introduced in Ref. 15, we define $b$ so that it reduces to $1 / l$ whenever the chain is unperturbed and tends to infinity whenever the chain attains full extension under the action of large forces. An expression for $b$ that satisfies these constraints is given by

$$
b=\frac{1-\left\langle\mathbf{R}^{2}\right\rangle_{0} /\left\langle\mathbf{R}^{2}\right\rangle_{m}}{l\left[1-\left\langle\mathbf{R}^{2}\right\rangle /\left\langle\mathbf{R}^{2}\right\rangle_{m}\right]},
$$

where $\left\langle\mathbf{R}^{2}\right\rangle_{0}$ is the unperturbed mean square end-to-end distance of the chain, $\left\langle\mathbf{R}^{2}\right\rangle$ is the mean square end-to-end distance under the prevailing kinematic conditions, and $\left\langle\mathbf{R}^{2}\right\rangle_{m}$ is the mean square end-to-end distance at maximum extension. By using Eq. (1) to calculate $\left\langle\mathbf{R}^{2}\right\rangle$ in terms of $b$, it is possible to determine this quantity self-consistently from the solution of a nonlinear equation. This is the strategy that will be followed to calculate the mean fractional extension of the chain as a function of the Weissenberg number.

\section{DYNAMICS}

The dynamics of the above continuum finitely extensible nonlinear elastic chain can be described by the following Langevin equation: ${ }^{20,22}$

$$
\frac{\partial \mathbf{r}(\tau, t)}{\partial \tau}=g \mathbf{A} \cdot \mathbf{r}(\tau, t)+\frac{3 b}{\zeta} \frac{\partial^{2} \mathbf{r}(\tau, t)}{\partial \tau^{2}}+\boldsymbol{\theta}(\tau, t),
$$

where

$$
\mathbf{A}=\left(\begin{array}{lll}
0 & 1 & 0 \\
\alpha & 0 & 0 \\
0 & 0 & 0
\end{array}\right)
$$

is the velocity gradient tensor, $g$ is some measure of the strength of the flow, $\zeta$ is the monomer friction coefficient, and $\boldsymbol{\theta}(\tau, t)$ is a Gaussian white noise variable defined by a vanishing mean and a second moment given by

$$
\left\langle\theta_{\alpha}\left(\tau_{1}, t_{1}\right) \theta_{\beta}\left(\tau_{2}, t_{2}\right)\right\rangle=2 \zeta^{-1} \delta_{\alpha \beta} \delta\left(\tau_{1}-\tau_{2}\right) \delta\left(t_{1}-t_{2}\right) .
$$

The parameter $\alpha$ in the expression for the velocity gradient tensor $\mathbf{A}$ is a measure of the extent of deviation from simple shear flow, for which $\alpha=0$. In general, $\alpha$ lies between +1 and -1 , these extemes representing pure elongational and pure rotational flow, respectively. At intermediate values, the flow is of "mixed" type, and contains an excess or deficit of one or other of these two components.

The calculation of $\left\langle\mathbf{R}^{2}\right\rangle$ is conveniently carried out in terms of the normal coordinates $\mathbf{X}_{p}(t)$ defined by ${ }^{20,23}$

$$
\mathbf{X}_{p}(t)=\frac{L_{p}}{l} \int_{0}^{N} d \tau \mathbf{r}(\tau, t) \cos (p \pi \tau / N),
$$

where $L_{p}=\left(2-\delta_{p, 0}\right)^{1 / 2} \sqrt{l / N}$, with $\delta_{p, 0}$ being the Kronecker delta. A normal coordinate transformation of Eq. (3) is easily shown to produce the following equation for $\mathbf{X}_{p}(t)$ :

$$
\frac{d \mathbf{X}_{p}(t)}{d t}=g \mathbf{A} \cdot \mathbf{X}_{p}(t)-\frac{\lambda_{p}}{\zeta} \mathbf{X}_{p}(t)+\boldsymbol{\theta}_{p}(t),
$$

where

$$
\lambda_{p}=\frac{3 b p^{2} \pi^{2}}{N^{2}}
$$

and

$$
\boldsymbol{\theta}_{p}(t)=\frac{L_{p}}{l} \int_{0}^{N} d \tau \boldsymbol{\theta}(\tau, t) \cos (p \pi \tau / N) .
$$

By introducing the Laplace transform, defined by

$$
\tilde{F}(s)=\int_{0}^{\infty} d t F(t) e^{-s t},
$$

Eq. (7) can be converted to an algebraic equation, which is easily solved to produce

$$
\tilde{\mathbf{X}}_{p}(s)=\mathbf{P}_{p}^{-1}(s) \cdot \mathbf{u}_{p}(s),
$$

where $\mathbf{u}_{p}(s)=\mathbf{X}_{p}(0)+\tilde{\boldsymbol{\theta}}_{p}(s)$, and 


$$
\mathbf{P}_{p}(s)=\left(\begin{array}{ccc}
a_{p}(s) & -g & 0 \\
-g \alpha & a_{p}(s) & 0 \\
0 & 0 & a_{p}(s)
\end{array}\right)
$$

with $a_{p}(s)=s+\lambda_{p} / \zeta$, and $\mathbf{X}_{p}(0)$ the initial value of the normal coordinate $\mathbf{X}_{p}(t)$. The inverse of the matrix $\mathbf{P}_{p}(s)$ is readily calculated as

$$
\begin{aligned}
\mathbf{P}_{p}^{-1}(s)= & \frac{1}{a_{p}^{3}(s)-g^{2} \alpha a_{p}(s)} \\
& \times\left(\begin{array}{ccc}
a_{p}^{2}(s) & g a_{p}(s) & 0 \\
g \alpha a_{p}(s) & a_{p}^{2}(s) & 0 \\
0 & 0 & a_{p}^{2}(s)-g^{2} \alpha
\end{array}\right) .
\end{aligned}
$$

The mean square end-to-end distance at time $t,\left\langle\mathbf{R}^{2}(t)\right\rangle$, can be obtained from the normal coordinates $\mathbf{X}_{p}(t)$ using the relation

$$
\begin{aligned}
\left\langle\mathbf{R}^{2}(t)\right\rangle & =\frac{8 l}{N} \sum_{p, q \text { odd }}\left\langle\mathbf{X}_{p}(t) \cdot \mathbf{X}_{q}(t)\right\rangle \\
& =\frac{8 l}{N} \sum_{p, q \text { odd }}\left\langle\mathbf{X}_{p}\left(t_{1}\right) \cdot \mathbf{X}_{q}\left(t_{2}\right)\right\rangle_{t_{2} \rightarrow t_{1}=t} .
\end{aligned}
$$

The average in the second line of the above equation can be re-expressed as an average involving Laplace variables,

$$
\left\langle\mathbf{X}_{p}\left(t_{1}\right) \cdot \mathbf{X}_{q}\left(t_{2}\right)\right\rangle=\mathcal{L}_{s_{1}}^{-1} \mathcal{L}_{s_{2}}^{-1}\left\langle\tilde{\mathbf{X}}_{p}\left(s_{1}\right) \cdot \tilde{\mathbf{X}}_{q}\left(s_{2}\right)\right\rangle,
$$

where $\mathcal{L}_{s_{1}}^{-1}$ and $\mathcal{L}_{s_{2}}^{-1}$ denote the inverse Laplace transforms with respect to the variables $s_{1}$ and $s_{2}$, respectively, which are the Laplace variables conjugate to the times $t_{1}$ and $t_{2}$. By combining Eq. (11) with Eq. (13), one finds that the average $\left\langle\widetilde{\mathbf{X}}_{p}\left(s_{1}\right) \cdot \widetilde{\mathbf{X}}_{q}\left(s_{2}\right)\right\rangle$ can be written in the general form,

$$
\begin{aligned}
\left\langle\tilde{\mathbf{X}}_{p}\left(s_{1}\right) \cdot \tilde{\mathbf{X}}_{q}\left(s_{2}\right)\right\rangle= & \sum_{\alpha=x, y, z} T_{p}^{(\alpha)}\left(s_{1}\right) T_{q}^{(\alpha)}\left(s_{2}\right) \\
& \times\left\{\left\langle X_{p}^{(\alpha)}(0) X_{q}^{(\alpha)}(0)\right\rangle\right. \\
& \left.+\left\langle\tilde{\theta}_{p}^{(\alpha)}\left(s_{1}\right) \tilde{\theta}_{q}^{(\alpha)}\left(s_{2}\right)\right\rangle\right\},
\end{aligned}
$$

where $T_{p}^{(\alpha)}\left(s_{1}\right)$ and $T_{q}^{(\alpha)}\left(s_{2}\right)$ are known functions of the Laplace variables $s_{1}$ and $s_{2}$ and the normal mode indices $p$ and $q$. The inverse Laplace transforms of these functions are easily calculated; they involve decaying exponentials in the times $t_{1}$ or $t_{2}$. The inverse transforms of the functions $T_{p}^{(\alpha)}\left(s_{1}\right) \widetilde{\theta}_{p}^{(\alpha)}\left(s_{1}\right)$ and $T_{q}^{(\alpha)}\left(s_{2}\right) \tilde{\theta}_{p}^{(\alpha)}\left(s_{2}\right)$ can be calculated from the convolution theorem, and the equation

$$
\left\langle\tilde{\theta}_{p}^{(\alpha)}\left(t_{1}\right) \tilde{\theta}_{q}^{(\alpha)}\left(t_{2}\right)\right\rangle=\frac{N}{\zeta} \frac{L_{p} L_{q}}{l^{2}} \delta\left(t_{1}-t_{2}\right) \delta_{p, q} ;
$$

they involve combinations of exponentials in $t_{1}$ or $t_{2}$ and various time-independent factors. These results are all substituted into Eq. (15) for the end-to-end distance, the times $t_{1}$ and $t_{2}$ being set to $t$ according to the prescription indicated there. Finally, since we are interested in results in the steady state (ss), the limit $t \rightarrow \infty$ is taken, whereupon all contribu- tions involving the initial state $\left\langle X_{p}^{(\alpha)}(0) X_{q}^{(\alpha)}(0)\right\rangle$ are eliminated (by virtue of the exponentially decaying coefficients that multiply them), and one is left with

$$
\left\langle\mathbf{R}^{2}\right\rangle_{\mathrm{ss}}=\frac{8}{N} \sum_{p}\left[\frac{3}{\lambda_{p}}+\frac{g^{2} \zeta^{2}(1+\alpha)^{2}}{2 \lambda_{p}\left(\lambda_{p}^{2}-g^{2} \zeta^{2} \alpha\right)}\right] .
$$

It is easily verified that when $\alpha=0$ (i.e., when the flow corresponds to simple shear) Eq. (19) above reduces to the result we had obtained in Ref. 15. (That result was obtained using a Green's function approach; the present approach, using the Langevin equation and a normal mode decomposition is somewhat simpler to implement.)

If we now introduce into Eq. (19) the definitions $\tau$ $=\zeta N^{2} l^{2} / 3 \pi^{2}$ for the longest relaxation time $\tau^{20} \mathrm{Wi}=g \tau$ for the Weissenberg number, ${ }^{1} \beta=\left\langle\mathbf{R}^{2}\right\rangle_{0} /\left\langle\mathbf{R}^{2}\right\rangle_{m}$ for the ratio of the unperturbed to maximum mean square end-to-end distances, and $z=\left\langle\mathbf{R}^{2}\right\rangle /\left\langle\mathbf{R}^{2}\right\rangle_{m}$ for the square of the mean fractional extension, Eq. (19) is transformed to

$$
\frac{z}{\beta}=\frac{1-z}{1-\beta}+\frac{4}{3 \pi^{2}}\left(\frac{1-z}{1-\beta}\right)^{3} \mathrm{Wi}^{2}(1+\alpha)^{2} S,
$$

where $S$ is the sum

$$
S=\sum_{p \text { odd }} \frac{1}{p^{6}-\alpha \mathrm{Wi}^{2} p^{2}(1-z)^{2} /(1-\beta)^{2}} .
$$

This sum can be calculated analytically, either using Mathematical or using results tabulated in Ref. 24. The result is

$$
\begin{aligned}
S= & -\frac{\pi^{2}}{8 \alpha \mathrm{Wi}^{2}}\left(\frac{1-\beta}{1-z}\right)^{2}+\frac{\pi}{8\left(\alpha \mathrm{Wi}^{2}\right)^{5 / 4}}\left(\frac{1-\beta}{1-z}\right)^{5 / 2} \\
& \times\left[\tan \left(\frac{\pi}{2}\left(\alpha \mathrm{Wi}^{2}\right)^{1 / 4}\left(\frac{1-\beta}{1-z}\right)^{1 / 2}\right)\right. \\
& \left.+\tanh \left(\frac{\pi}{2}\left(\alpha \mathrm{Wi}^{2}\right)^{1 / 4}\left(\frac{1-\beta}{1-z}\right)^{1 / 2}\right)\right] .
\end{aligned}
$$

Equation (20) along with Eq. (22) are the principal results of this paper. They provide an implicit expression for the fractional extension of the chain $x$, which we define as $x=\sqrt{z}$, in terms of the Weissenberg number Wi and the mixing parameter $\alpha$. The calculation of $x$ can only be carried out numerically, but the calculation is relatively straightforward. The results are described in the next section.

\section{RESULTS AND DISCUSSION}

Figure 1 compares our theoretical results for the mean fractional extension against results from the Brownian dynamics simulations of Chu et al. on a 150-bead Kramer's chain, ${ }^{12}$ the raw data for which were communicated to us privately. The figure shows $x$ vs Wi for six different fixed values of the parameter $\alpha$. The solid lines are the theoretical curves as calculated from Eq. (20) using the definition $x$ $=\sqrt{z}$, and the symbols are data points from the simulations. The topmost curve corresponds to purely extensional flow [i.e., flow in which the amount of the extensional component is $100 \%$, or $100 \% E$ in the notation used in the figure and in Ref. 12, the relation between $\% E$ and $\alpha$ being given by 


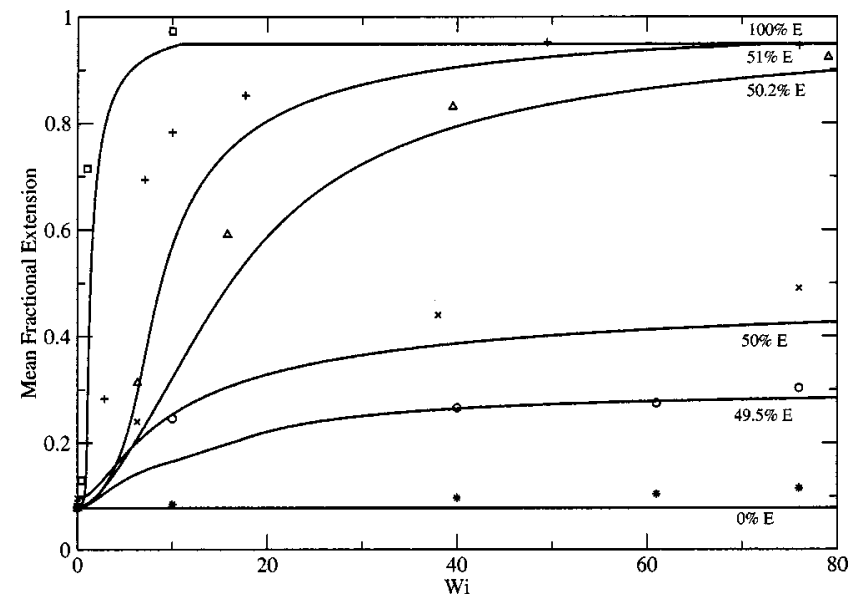

FIG. 1. Mean fractional extension $x \equiv\left\langle\mathbf{R}^{2}\right\rangle^{1 / 2} / N$ vs Weissenberg number Wi for different fixed values of the mixing parameter $\alpha$. The full lines are the theoretical curves calculated from Eqs. (20) and (22). The symbols are data points from the simulations of Ref. 12. The topmost curve corresponds to pure extensional flow $(100 \% E)$ and the bottom most curve to pure rotational flow $(0 \% E)$. The intermediate curves correspond to flows with intermediate amounts of the extensional component, as indicated on the curve. The curve with $E=50 \%$ describes simple shear. The symbols have the following meanings: open squares, $100 \% E$; plus signs, $51 \% E$; open triangles, $50.2 \% E$; crosses, $50 \% E$; open circles, $49.5 \% E$; asterisks, $0 \% E$.

$\% E=50(1+\alpha)]$. The open squares are the data points from the simulation with this value of $\alpha$. The bottom most curve corresponds to purely rotational flow $(0 \% E)$, and the corresponding data from the simulation are represented by asterisks. The curves in between correspond to flows with $49.5 \%$, $50 \%, 50.2 \%$, and $51 \%$ of the extensional component, the simulation data for these flows being represented, respectively, by open circles, crosses, open triangles, and plus signs. The simulation data in Fig. 1 are essentially a reproduction of Fig. 3(a) in Ref. 12.

In constructing the theoretical curves using Eq. (20), a definite value has to be assigned to the parameter $\beta$, which we have done as follows. Given that $\beta$ is the ratio of the mean square end-to-end distances of the unperturbed chain and the chain at maximum extension, it may be rewritten equivalently as, $\beta=\left\langle\mathbf{R}^{2}\right\rangle_{0} / N^{2} /\left\langle\mathbf{R}^{2}\right\rangle_{m} / N^{2} \equiv z_{\min }^{2} / z_{\max }^{2}$. The parameters $z_{\min }$ and $z_{\max }$ are estimated from the simulation data of $x$ versus $\mathrm{Wi}$ in the limits $\mathrm{Wi}=0$ (for $z_{\min }$ ) and $\mathrm{Wi} \rightarrow \infty$ (for $\left.z_{\max }\right)$. The values so determined for the pair $\left(z_{\min }, z_{\max }\right)$ for the flow types corresponding to $100 \% E, 51 \% E, 50.2 \% E$, $50 \% E, 49.5 \% E$, and $0 \% E$ are, respectively, $(0.08,1.0)$, $(0.081,1.0), \quad(0.078,1.0), \quad(0.0961,0.5), \quad(0.0791,0.3)$, and $(0.078,0.1)$. In the case of pure rotational flow, $z_{\max }$ can actually be assigned any value greater than $z_{\min }$ and less than 1.0 without affecting the results.

Although the theoretical curves in Fig. 1 are not in quantitative agreement with the data from the simulation, they do reproduce the overall trends. In particular, they correctly predict the almost instantaneous stretching to full extension of such molecules in purely extensional flow, and the lack of any stretching whatever in purely rotational flow. In the case of simple shear $(50 \% E)$, they also correctly reproduce the experimental observation, obtained on single molecule samples of flexible DNA, of close to $50 \%$ full extension at

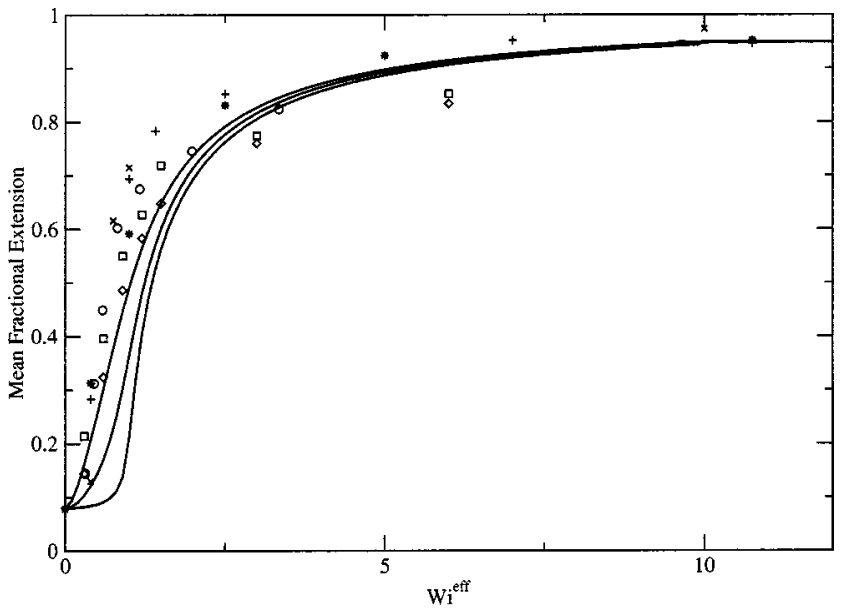

FIG. 2. Mean fractional extension $x$ vs the scaling variable $\mathrm{Wi}^{\mathrm{eff}}=\sqrt{\alpha} \mathrm{Wi}$ for $\alpha>0$. The full lines are the theoretical curves calculated from Eqs. (20) and (22). The symbols represent data points from the simulations of Ref. 12 and from the unpublished experiments of Babcock et al.

Weissenberg numbers of about 80 . For all the flow types, the greatest deviations between theory and simulation occur at the smallest Wi values, and are similar, qualitatively, to the low Wi deviations seen earlier in comparisons of the model with experimental measurements of DNA in simple shear. ${ }^{15,16}$ The neglect of hydrodynamic interactions may be a contributing factor to the behavior seen in this regime; such interactions, if they do indeed play a role, would be most important here. At higher Wi, when the chains are more extended, their importance, can be expected progressively to diminish. But even at low Wi, it is not clear that hydrodynamic interactions contribute significantly to the chain dynamics. A calculation by Wang and Chatterjee ${ }^{25}$ on polymer behavior in flow, in which these interactions are treated approximately in preaveraged fashion, leads to only modest improvement over the results of the Rouse model in comparisons with experiments. More recent stochastic simulations by Graham et al. ${ }^{11}$ on the flow behavior of $\lambda$-phage DNA suggest that both free draining and nonfree draining models of the chain are almost equally successful in reproducing experimental trends for stretching under shear and extension. The inclusion of hydrodynamic interactions in the present model would provide a further test of their importance to chain dynamics under flow, but the necessary calculations are quite complex, and will form the subject of a future publication.

Figure 2 is a test of the suggestion made in Ref. 12 that the parameter $\mathrm{Wi}^{\mathrm{eff}}=\sqrt{\alpha} \mathrm{Wi}$ constitutes a scaling variable in terms of which the data of $x$ versus Wi fall on a single universal curve. The figure shows simulation data from Ref. 12, unpublished experimental data by Babcock et al., and the theoretical curves derived from Eq. (20), all the results referring to the case $\alpha>0$. The full lines are the theoretical curves, and the symbols are simulation and experimental data points. The experimental and simulation data in this figure are essentially a reproduction of Fig. 3(b) in Ref. 12. It is clear from the figure that there is a significant degree of data collapse when $\mathrm{Wi}^{\mathrm{e}}$ eff is used to plot the data, both for the experimental and simulation results as well as for the theo- 
retical results. An inspection of Eq. (20) shows why $\mathrm{Wi}^{\mathrm{eff}}$ is a reasonably effective scaling variable; $\alpha$ and Wi appear $a l$ most everywhere in this equation as the combination $\sqrt{\alpha}$ Wi. Because they do not appear everywhere in this combination, however, data collapse is not expected to be perfect, as indeed it is not. Again, it is the low Wi regions of the theoretical curves that exhibit the most marked departures from universality. Why this should be so is not clear.

We have not been able to see evidence in our calculations of similar scaling relations for the case $\alpha<0$; apparently, neither have Chu and his co-workers. At present we do not know why.

From all of the above results, it is clear that the finitely extensible Rouse model introduced earlier is not restricted to the description of polymers in simple shear alone, but can be used to provide a reasonably accurate description of polymers in mixed flows as well. Our calculations with this model (both the present one and the earlier one) suggest that it is the proper treatment of the effects of finite extensibility that ensures that the model does not fail under conditions of strong flow. As we have seen earlier, the neglect of the finite extensibility constraint can produce unphysical effects under these conditions. However, a purely Gaussian treatment of chain statistics may be adequate if the flow is weak and the extent of chain deformation therefore small; in this limit, the chain distribution function remains Gaussian, as shown by Takserman-Kozer ${ }^{26}$ in an early discussion of the mixed flow problem, but even in this limit it is not a simple superposition of effects arising from the individual elongational and rotational components of the flow.

\section{ACKNOWLEDGMENTS}

We are grateful to Steven Chu and Joe S. Hur of Stanford University for providing us with raw data from their simulations of chain extension in mixed flows. A. D. acknowledges financial support from the Max Planck Institute, Germany.
${ }^{1}$ D. E. Smith, H. P. Babcock, and S. Chu, Science 283, 1724 (1999).

${ }^{2}$ P. LeDuc, C. Haber, G. Bao, and D. Wirtz, Nature (London) 399, 564 (1999).

${ }^{3}$ B. Ladoux and P. S. Doyle, Europhys. Lett. 52, 511 (2000).

${ }^{4}$ P. S. Doyle, B. Ladoux, and J.-L. Viovy, Phys. Rev. Lett. 84, 4769 (2000).

${ }^{5}$ T. T. Perkins, D. E. Smith, and S. Chu, Science 276, 2016 (1997); D. E. Smith and S. Chu, ibid. 281, 1335 (1998); H. P. Babcock, D. E. Smith, J. S. Hur, E. S. G. Shaqfeh, and S. Chu, Phys. Rev. Lett. 85, 2018 (2000); J. S. Hur, E. S. G. Shaqfeh, H. P. Babcock, D. E. Smith, and S. Chu, J. Rheol. 45, 421 (2001)

${ }^{6}$ P.-G. de Gennes, J. Chem. Phys. 60, 5030 (1974).

${ }^{7}$ T. W. Liu, J. Chem. Phys. 90, 5826 (1989).

${ }^{8}$ J. J. López Cascales and J. García de la Torre, Polymer 32, 3359 (1991); J. J. López Cascales, F. G. Díaz, and J. García de la Torre, ibid. 36, 345 (1995); J. J. López Cascales, S. Navarro, and J. García de la Torre, Macromolecules 25, 3574 (1992).

${ }^{9}$ P. S. Doyle, E. S. G. Shaqfeh, and A. P. Gast, J. Fluid Mech. 334, 251 (1997); N. C. Andrews, A. J. Mchugh, and J. D. Schieber, J. Rheol. 42, 281 (1998); J. S. Hur, E. S. G. Shaqfeh, and R. G. Larson, ibid. 44, 713 (2000).

${ }^{10}$ A. V. Lyulin, D. B. Adolf, and G. R. Davies, J. Chem. Phys. 111, 758 (1999).

${ }^{11}$ R. M. Jendrajack, J. J. de Pablo, and M. D. Graham, J. Chem. Phys. 116, 7752 (2002).

${ }^{12}$ J. S. Hur, E. S. G. Shaqfeh, H. P. Babcock, and S. Chu, Phys. Rev. E 66, 011915 (2002).

${ }^{13}$ G. G. Fuller and L. G. Leal, Rheol. Acta 19, 580 (1980).

${ }^{14}$ D. J. Amit, Field Theory, Renormalization Group and Critical Phenomena (McGraw-Hill, New York, 1978).

${ }^{15}$ A. Dua and B. J. Cherayil, J. Chem. Phys. 112, 8707 (2000).

${ }^{16}$ A. Dua and B. J. Cherayil, J. Chem. Phys. 113, 10776 (2000).

${ }^{17}$ B. Gaveau and L. S. Schulman, Phys. Rev. A 42, 3470 (1990).

${ }^{18}$ R. B. Bird, C. F. Curtiss, R. C. Armstrong, and O. Hassager, Dynamics of Polymeric Liquids (Wiley, New York, 1987), Vol. 2.

${ }^{19}$ K. F. Freed, Adv. Chem. Phys. 22, 1 (1972); N. Saitô, K. Takahashi, and Y. Yunoki, J. Phys. Soc. Jpn. 22, 219 (1967).

${ }^{20}$ M. Doi and S. F. Edwards, The Theory of Polymer Dynamics (Oxford, New York, 1986).

${ }^{21}$ K. F. Freed, Renormalization Group Theory of Macromolecules (Wiley, New York, 1987).

${ }^{22}$ S.-Q. Wang and K. F. Freed, J. Chem. Phys. 88, 3944 (1988).

${ }^{23}$ S.-Q. Wang and K. F. Freed, J. Chem. Phys. 85, 6210 (1986).

${ }^{24}$ E. R. Hansen, A Table of Series and Products (Prentice-Hall, New Jersey, 1975).

${ }^{25}$ X. Wang and A. P. Chatterjee, Macromolecules 34, 1118 (2001).

${ }^{26}$ R. Takserman-Krozer, J. Polym. Sci., Part C: Polym. Symp. 16, 2845 (1967); 16, 2855 (1967). 